\title{
Evidence for cognitive vestibular integration impairment in idiopathic scoliosis patients Martin Simoneau ${ }^{* 1,2}$, Vincent Lamothe ${ }^{1}$, Émilie Hutin ${ }^{1}$, Pierre Mercier ${ }^{1}$, Normand Teasdale $e^{1,2}$ and Jean Blouin ${ }^{3}$
}

Address: ${ }^{1}$ Faculté de Médecine, Université Laval, Québec, Canada, ${ }^{2}$ Centre de recherche sur le vieillissement de Québec du Centre de recherche FRSQ Hôpital Saint-Sacrement, Québec, Canada and ${ }^{3}$ Neurobiology and Cognition Laboratory, CNRS and Aix Marseille University, Marseille, France

Email: Martin Simoneau* - Martin.Simoneau@kin.msp.ulaval.ca; Vincent Lamothe - pedalos@ hotmail.com;

Émilie Hutin - emilie.hutin@hmn.aphp.fr; Pierre Mercier - eburn@videotron.ca; Normand Teasdale - Normand.Teasdale@kin.msp.ulaval.ca; Jean Blouin - Jean.Blouin@univ-provence.fr

* Corresponding author

Published: 25 August 2009

BMC Neuroscience 2009, 10:102 doi:10.1/86/1471-2202-10-102
Received: 3 April 2009

Accepted: 25 August 2009

This article is available from: http://www.biomedcentral.com/I47I-2202/10/102

(c) 2009 Simoneau et al; licensee BioMed Central Ltd.

This is an Open Access article distributed under the terms of the Creative Commons Attribution License (http://creativecommons.org/licenses/by/2.0), which permits unrestricted use, distribution, and reproduction in any medium, provided the original work is properly cited.

\begin{abstract}
Background: Adolescent idiopathic scoliosis is characterized by a three-dimensional deviation of the vertebral column and its etiopathogenesis is unknown. Various factors cause idiopathic scoliosis, and among these a prominent role has been attributed to the vestibular system. While the deficits in sensorimotor transformations have been documented in idiopathic scoliosis patients, little attention has been devoted to their capacity to integrate vestibular information for cognitive processing for space perception. Seated idiopathic scoliosis patients and control subjects experienced rotations of different directions and amplitudes in the dark and produced saccades that would reproduce their perceived spatial characteristics of the rotations (vestibular condition). We also controlled for possible alteration of the oculomotor and vestibular systems by measuring the subject's accuracy in producing saccades towards memorized peripheral targets in absence of body rotation and the gain of their vestibulo-ocular reflex.

Results: Compared to healthy controls, the idiopathic scoliosis patients underestimated the amplitude of their rotations. Moreover, the results revealed that idiopathic scoliosis patients produced accurate saccades to memorized peripheral targets in absence of body rotation and that their vestibulo-ocular reflex gain did not differ from that of control participants.

Conclusion: Overall, results of the present study demonstrate that idiopathic scoliosis patients have an alteration in cognitive integration of vestibular signals. It is possible that severe spine deformity developed partly due to impaired vestibular information travelling from the cerebellum to the vestibular cortical network or alteration in the cortical mechanisms processing the vestibular signals.
\end{abstract}




\section{Background}

Idiopathic scoliosis is a complex deformation of the spine and it is the most common type of spine deformity. Its prevalence is about $2 \%$ to $3 \%$ in children aged between 10 to 16 years old and girls are at a higher risk than boys for severe progression. Although the etiopathogenesis of scoliosis is unknown, various factors have been identified that could be related to the etiology of scoliosis. Among these factors, it has been proposed that neurological mechanisms could be related to scoliosis [1]. Nonetheless, no clear-cut neurological tests either for diagnosing idiopathic scoliosis or for predicting its progression have so far been established.

Neurological deficits in idiopathic scoliosis have mostly been evidenced by studies examining the patient's motor behavior. For instance, several studies have demonstrated that compared to age-matched individuals, idiopathic scoliosis patients (ISP) show deficits in controlling their postural sway [e.g., [2]]. Because many idiopathic scoliosis patients show longer somatosensory cortical potentials (parietal N37) compared to healthy individuals, their balance instabilities could be associated with alteration in sensory signal processing [3-5]. Consistent with this hypothesis, impairment in balance control has been observed in ISP when sensory information is restored following transient deprivation of visual or somatosensory information (through vibration of the muscles around the ankle joint) [6]. The fact that postural sway increased when sensory information returned to normal leads to the suggestion that ISP have difficulty in dynamically adjusting the weight of the various sensory inputs to tailor the balance control commands.

Cases have been reported suggesting that the motor deficits in ISP may also be related to alterations in processing vestibular signals and in transforming these signals into motor commands. For instance, when the vestibular signals remain the only valuable source of information (i.e., in absence of vision and when ankle proprioception is altered through tendon vibration), body sway of ISP is aggravated compared to control participants [7-10]. These observations are in agreement with those published by Gauchard's et al. [11] revealing that ISP failed the balance control tasks that challenge the vestibular system.

While the deficits in sensorimotor transformations have been documented in ISP, little attention has been devoted to their capacity to integrate vestibular information for cognitive processing. According to electrophysiological and neuroimaging studies, the output of the vestibular apparatus projects either directly or indirectly to cortical regions of the brain essential for spatial processing (e.g., parietal cortex [12]). Furthermore, behavioral experiments conducted on healthy and neurological subjects have shown that perception of self movement and spatial orientation greatly rely on vestibular signal processing [13-16].

In addition to their documented deficits in transforming sensory signals into proper motor commands (e.g., $[3,7,8,10])$, here we tested whether ISP also show impairments in the processing of vestibular signals for cognitive spatial processes.

\section{Results}

The analysis of the cognitive vestibular gain (Fig. 1 upper panel) showed that both groups underestimated the whole-body rotation as demonstrated by their average which are below unity. Nonetheless, the ISP underestimated the magnitude of the whole-body rotation to a greater extent than the control participants (mean of 0.65 and 0.82 for ISP and Control, respectively; main effect of Group; $\left.\mathrm{F}_{1,20}=5.57, \mathrm{p}<0.05\right)$. The absence of significant interactions (i.e., Group by Direction or Group by Direction by Amplitude; ps > 0.05) suggests that, for both groups, the cognitive vestibular gains did not depend on the directions of the chair rotation nor on their amplitudes. On the other hand, the saccades produced by the ISP in the absence of whole-body rotation (Fig. 1 lower panel) were accurate and their amplitude was not significantly different to that of control participants $(\mathrm{t}=1.88$, df $=20, \mathrm{p}>0.05$ ).

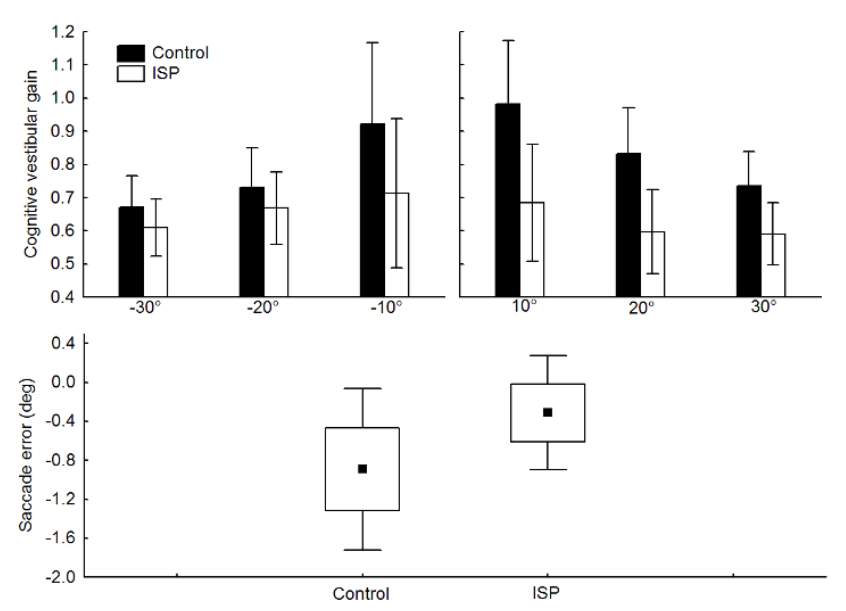

\section{Figure I}

experimental results. Upper panel depicts both groups mean vestibular cognitive gain for chair rotations of $\pm 10^{\circ}, \pm$ $20^{\circ}$ and $\pm 30^{\circ}$. The error bars represent $\pm 95 \%$ confidence interval. Lower panel presents both group means (black square $=$ mean, boxes $=$ standard error and, error bars $=$ $1.96 \times$ standard error) for the saccade accuracy in absence of chair rotation. Data for the $\pm 15^{\circ}$ targets were pooled as no significant effect of direction was observed. 
The analyses revealed that the VOR gain (all participants results appear in table 1 ) of the ISP did not differ from that of control participants regardless of the rotation direction (no main effect of Group or Direction and no Group by Direction interaction: ps > 0.05). Importantly, the high percentage of the variance explained by the linear regression model for all participants confirms the efficiency of the model in quantifying the gain of the VOR for all participants and both rotations (no main effect of Group, Direction and no Group by Direction interaction: ps $>0.05)$.

\section{Discussion}

The present study confirms previous results revealing a good capacity of the control subjects to memorize and process vestibular signals to determine whole-body motions [13,15-21]. Compared to controls, however, the ISP underestimated the amplitude of the rotations suggesting impairment in their ability to memorize and process vestibular signals. None of the ISP showed asymmetry in perceiving leftward and rightward rotations. It is noteworthy that in absence of body rotation, ISP performed accurate saccades to memorized targets excluding any saccadic motor deficit. This observation substantiates previous results suggesting that the network assisting the control of vestibular memory-guided saccades is different from the one governing visual memory-guided saccades $[22,23]$. Moreover, the fact that ISP had normal vestibuloocular reflex gain confirms normal vestibular system in these patients.

Using the magnetic resonance imaging technique, Liu et al. [24] have recently demonstrated that the volume of several anatomical brain regions of ISP differs with respect to controls. For instance, compared to controls, ISP have larger parietal, temporal and frontal lobes. Despite the fact that no clear link can be established between brain volume and dysfunction, it is worth mentioning that these brain regions receive vestibular inputs and are involved in spatial attention and processing $[12,13,22]$. Therefore, the impairment of the ISP to memorize and process vestibular inputs could be due to an alteration of the cortical network processing vestibular signals. This would be consistent with the findings that, repetitive tran-

Table I: Gain of the vestibulo-ocular reflex and variance explained.

\begin{tabular}{|c|c|c|c|c|c|}
\hline \multirow[b]{2}{*}{ ID } & \multirow[b]{2}{*}{ Group } & \multicolumn{2}{|c|}{ ROTATION + $20^{\circ}$} & \multicolumn{2}{|c|}{ ROTATION $-20^{\circ}$} \\
\hline & & $\begin{array}{l}\text { VOR } \\
\text { gain }\end{array}$ & Variance explained & VOR gain & Variance explained \\
\hline 1 & Control & 0.92 & 0.90 & 0.91 & 0.88 \\
\hline 2 & Control & 1.11 & 0.92 & 0.99 & 0.90 \\
\hline 3 & Control & 0.92 & 0.92 & 0.83 & 0.79 \\
\hline 4 & Control & 1.04 & 0.97 & 1.07 & 0.97 \\
\hline 5 & Control & 1.00 & 0.96 & 0.93 & 0.92 \\
\hline 6 & Control & 0.89 & 0.92 & 0.92 & 0.90 \\
\hline 7 & Control & 1.03 & 0.99 & 1.07 & 0.97 \\
\hline 8 & Control & 0.94 & 0.93 & 0.94 & 0.91 \\
\hline 9 & Control & 1.19 & 0.96 & 1.13 & 0.95 \\
\hline 10 & Control & 1.13 & 0.93 & 1.14 & 0.95 \\
\hline II & Control & 1.08 & 0.96 & 1.09 & 0.94 \\
\hline \multirow[t]{2}{*}{12} & Control & 0.97 & 0.87 & 1.02 & 0.93 \\
\hline & Mean & 1.02 & 0.94 & 1.00 & 0.92 \\
\hline 1 & ISP & 1.03 & 0.95 & 0.97 & 0.93 \\
\hline 2 & ISP & 1.06 & 0.93 & 1.08 & 0.91 \\
\hline 3 & ISP & 1.00 & 0.97 & 1.10 & 0.97 \\
\hline 4 & ISP & 1.03 & 0.92 & 0.89 & 0.89 \\
\hline 5 & ISP & 0.87 & 0.85 & 1.07 & 0.90 \\
\hline 6 & ISP & 0.98 & 0.95 & 1.10 & 0.94 \\
\hline 7 & ISP & 0.93 & 0.91 & 1.01 & 0.95 \\
\hline 8 & ISP & 1.08 & 0.93 & 1.06 & 0.96 \\
\hline 9 & ISP & 0.98 & 0.98 & 0.98 & 0.97 \\
\hline \multirow[t]{2}{*}{10} & ISP & 0.94 & 0.89 & 0.99 & 0.97 \\
\hline & Mean & 0.99 & 0.93 & 1.02 & 0.94 \\
\hline
\end{tabular}

Note: for technical reason, we could not quantify the gain of the VOR of one control participant. 
scranial magnetic stimulation applied to the right posterior parietal cortex of healthy participants, impairs encoding of vestibular-derived displacement perception [25].

In addition, as idiopathic scoliosis is associated with melatonin signaling deficit [26], ISP impairment in perceiving whole-body rotation may witness alteration of the vestibulocerebellum functions. Indeed, although the human cerebellum is not considered as an endocrine system, the external zone of the cerebellar molecular layer has a high density of melatonin receptors [27]. Integrating vestibular, somatosensory and visual information, the vestibulocerebellum plays a key role in sensing head motion or head orientation in space and relative to gravity [28]. Remarkably, patients with midline cerebellar lesions underestimated the magnitude of their rotation despite normal vestibular function [29]. Therefore, it is likely that erroneous vestibular information going to the vestibular cortical network through cerebello-thalamocortical pathways prevents accurate computation of body rotation. However, not all processes mediated by the vestibulocerebellum would be altered as suggested by the ISP's accurate saccades to the memorized target in absence of body rotation. This may suggest that the rostral fastigial nuclei could be impaired by the melatonin signaling deficit in ISP. These nuclei, which are irresponsive to eye movements, are known as key processing centers for spatial orientation [30].

Manzoni and Miele [31] and Pompeiano et al. [32] have proposed that deficit in melatonin has an inhibitory effect on the vestibulospinal activity which could lead to abnormal activities of the cervical and axial muscles. In addition, impaired vestibular information from the cerebellum to the vestibular cortical network or alteration of this cortical network could also lead to alteration of back muscle activities. Therefore, vestibulospinal $[31,32]$ as well as corticospinal abnormal activities may be part of the mechanisms leading to the onset and progression of scoliosis.

\section{Conclusion}

The present results show that cognitive vestibular processing is impaired in ISP. It is possible that severe spine deformity developed partly due to impaired vestibular information travelling from the cerebellum to the vestibular cortical network or alteration in the cortical mechanisms processing of vestibular signals. The present study, however, does not allow one to make claims as to whether deficits in cognitive processing of vestibular signals should be considered as a potential factor leading to curve progression in scoliosis patients. Further studies may determine if this deficit and the melatonin signaling dys- function in ISP can predict curve progression in patients with small spine deformity.

\section{Methods \\ Subjects}

Ten idiopathic scoliosis patients $(9$ female, mean age $=$ 17.4, Cobb angle range $=2851$ ) and thirteen age-matched healthy individuals $(11$ female, mean age $=16.4)$ were tested and provided informed consent according to Laval University biomedical ethic committee. All the ISP had a right thoracic curve and a Riser sign greater than 3 . Their curve had not progressed during the last year and no ISP wore bracing. No ISP had clinically detectable neurological disorders nor history of operative treatment or trauma to the brain. Furthermore, all participants had normal or corrected-to-normal vision.

\section{Method and Procedure}

The participants sat in a completely dark room on a chair located in the center of a black cylinder with a radius of $1.5 \mathrm{~m}$. The subjects were secured to the chair using a fourpoint belt and a chin support preventing head movement on the trunk during the rotations. The chair was manually rotated around the vertical axis using the handle attached to the rear of the chair. The rotations were measured with a potentiometer fixed at the centre of rotation of the chair. An array of LEDs placed behind the chair was used to display the magnitude of the chair rotation to be produced by the experimenter for each trial. The pseudo-randomly selected magnitudes of the whole-body rotation were $10^{\circ}$, $20^{\circ}$ or $30^{\circ}$. The whole-body rotation was clockwise for half of the trials and counterclockwise for the other half. There were 8 trials for each rotation possibility. The chair was rotated following bell-shaped velocity profiles (Fig. 2) because it simulates the velocity profiles of natural head movements $[33,34]$. It is worth mentioning that similar whole-body dynamics were observed for both groups and direction (no main effects of Group or Direction and no Group by Direction by Amplitude interaction: ps > 0.05). As expected, however, the peak angular velocities were scaled according to the amplitude of the chair rotation $\left(\mathrm{F}_{2,40}=12.21, \mathrm{p}<0.001\right)$.

Horizontal eye movements were recorded using electrooculography (Biomedica Mangoni model BM623). Chair rotations and eye movements were monitored at $200 \mathrm{~Hz}$ using a 12 bits A/D board (model LabMaster, Scientific Solutions, Mentor, OH, USA).

The experimental session consisted in a main experimental condition that tested the ability of the participants to process vestibular inputs (i.e., cognitive vestibular condition) and two control conditions; one that examined subjects' capacity in producing accurate saccadic eye 


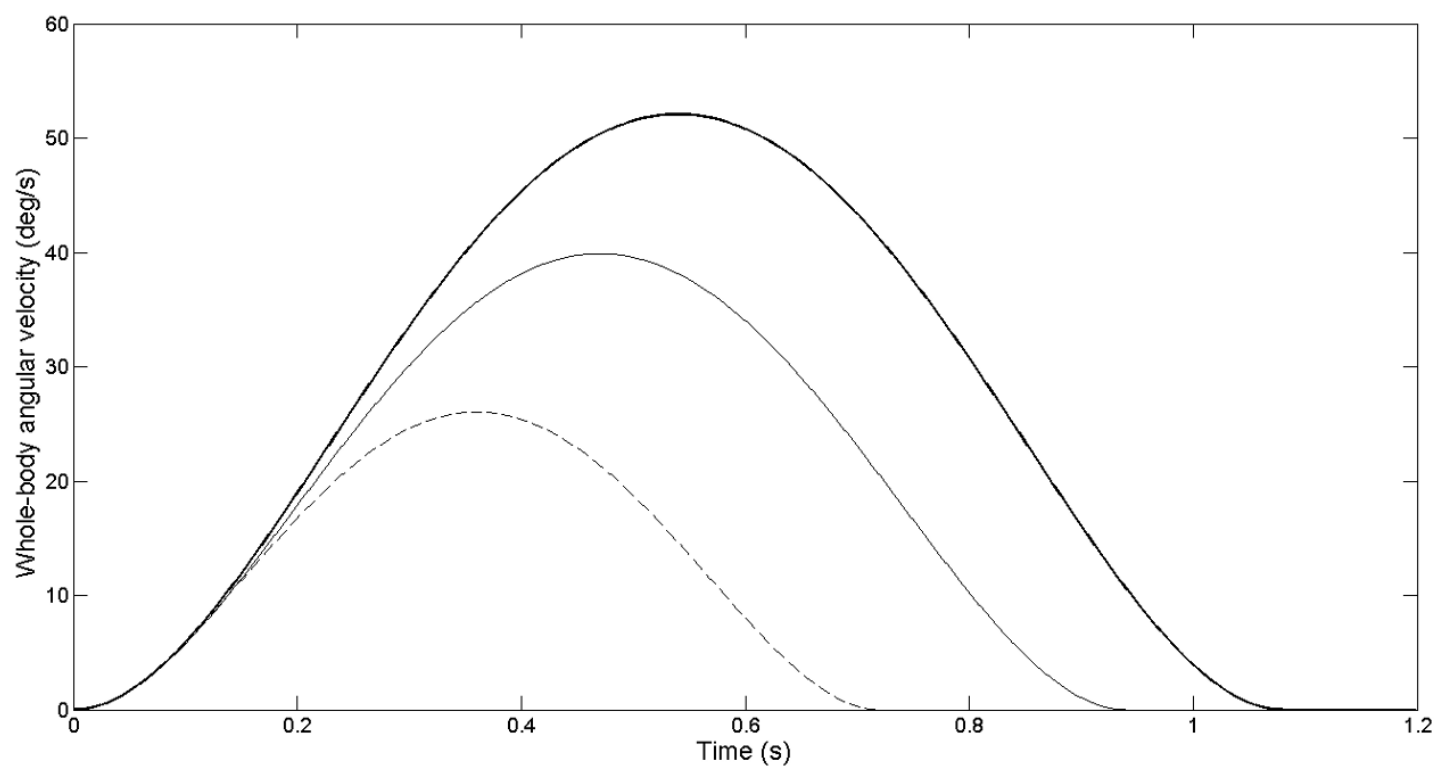

\section{Figure 2}

whole-body angular velocity: cognitive vestibular condition. Group mean time-series of the whole-body angular velocity for the chair rotation amplitude of $10^{\circ}$ (dashed line), $20^{\circ}$ (thin line) and $30^{\circ}$ (thick line) are presented. Only counter-clockwise rotations are illustrated. Data are for the scoliosis group.

movements (i.e., visual condition) and the other that controlled for possible alteration of the vestibular system (i.e., vestibulo-ocular reflex condition). In the cognitive vestibular condition, the participants were passively rotated. Eye movements were attenuated during body rotations by asking the subjects to look at a chair-fixed diode positioned straight-ahead. After the rotation, they produced a saccade in order to shift their gaze to their initial position ("vestibular memory-contingent saccade" paradigm [17]). The amplitude of the saccade (i.e., perceived body rotation amplitude) was measured at the offset of the first saccade (defined as the first instant that the eye velocity dropped below $5 \%$ after the saccade onset). Therefore, the participants had to process and memorize the vestibular signals generated during the whole-body rotation in order to produce a saccade of equal magnitude but in the opposite direction of the rotation (Fig. 3 upper panels). In the visual condition, we verified whether the generation of saccadic eye movements differed between control participants and ISP in the absence of whole-body rotation. We did this by measuring the saccades produced towards a memorized peripheral target set at $\pm 15^{\circ}$. In this condition, the participants looked at a diode located straight ahead (fixation diode) while the peripheral target lit during $200 \mathrm{~ms}$. Then, when the fixation diode was turned off (i.e., $1 \mathrm{~s}$ after the extinction of the peripheral target), the participants produced a saccade to the memo- rized visual target (Fig. 3 lower panels). Participants performed 10 trials for each target direction. We calculated the final angular error, that is, the angular difference between the memorized peripheral target and the final angular position of the gaze. As for the cognitive vestibular condition, the amplitude of the saccade was measured at the offset of the first saccade.

Herman et al. [1] reported that some ISP have qualitative asymmetrical vestibulo-ocular reflex gain suggesting that the vestibular system may be impaired in ISP. For this reason we also quantified the gain of the vestibulo-ocular reflex of our patients and control participants. The amplitude of the chair rotation was $\pm 20^{\circ}(10$ trials per direction). The chair angular velocity was bell-shaped and the peak angular velocities were similar for both directions and groups (no main effects of Group or Direction and no Group by Direction interaction, ps $>0.05$ ). The participants were instructed to keep their eyes on the fixation diode located straight ahead during the whole body rotation. For each trial, we calculated the gain of the vestibular-ocular reflex as the ratio between the slope of the linear regression of eye and chair velocities [35]. The fitting of the linear regression was performed from the onsets of the time-series of the eye angular velocity and the chair angular velocity until the peak eye angular velocity (i.e., group average: $208 \pm 0.021 \mathrm{~ms}$ and $215 \pm 0.028 \mathrm{~ms}$ for the ISP 


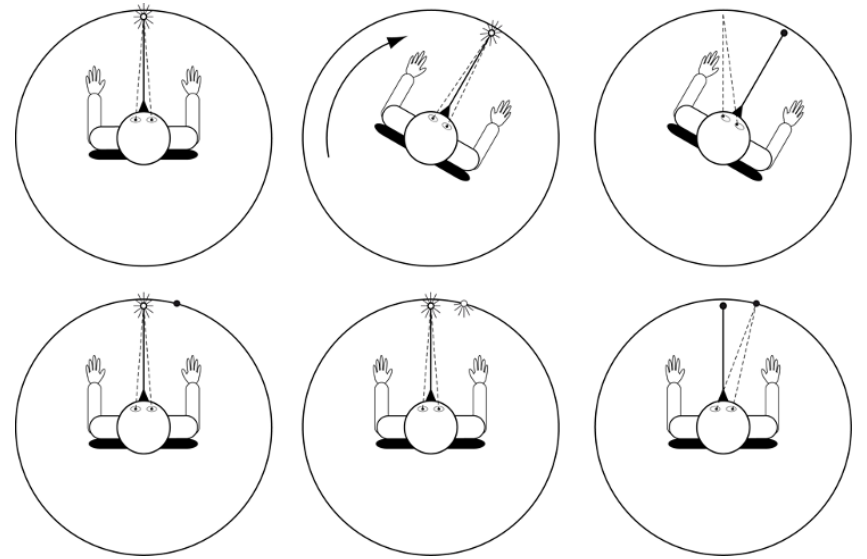

Figure 3

experimental conditions. Schematic of the experimental set-up: the upper panels depict the vestibular condition and lower panels the visual condition. Participants looked at a chair-fixed light (CFL) before being submitted to whole-body rotation (upper left panel). Then, the whole-body rotation stimulated mainly the horizontal semicircular canals (upper middle panel). Following the rotation, the CFL was turned off and the participants performed a saccade to the starting position (upper right panel). In the visual condition, the participants looked at the CFL (lower left panel). Then, the peripheral target lit up for $0.2 \mathrm{~s}$ (lower middle panel). Finally, following I s, the CFL was turned off and the participants performed a saccade to the peripheral target (lower right panel).

and control participants, respectively). To determine the accuracy of the fit of the linear regression, we calculated the coefficient of determination $\left(\mathrm{r}^{2}\right)$.

\section{Statistical analysis}

To quantify the cognitive vestibular gain, for each trial we divided the saccade amplitude (i.e., perceived angular rotation) by the actual whole-body rotation amplitude. Then, we performed a repeated-measures analysis of variance (Group by Amplitude by Direction). To ascertain that similar whole-body rotations were used for both groups in the cognitive vestibular condition, a repeatedmeasures analysis of variance (Group by Amplitude by Direction) was performed on the chair peak angular velocity. In the visual condition, we used a two-tailed ttest to verify if both groups produced different final saccadic angular error in absence of chair rotation. Finally, repeated-measures analyses of variance (Group by Direction) were used to assess the gain of the vestibulo-ocular reflex and the goodness of fit (i.e., variance explained by the model) of the linear regression model. All results were considered to be significant at the 5\% critical level ( $\mathrm{p}<$ 0.05).

\section{Competing interests}

The authors declare that they have no competing interests.

\section{Authors' contributions}

MS, NT and JB conceived the study. PM recruited the subjects and examined all the patients. VL and EH performed the data acquisition and analyses. EH and MS developed the Matlab programs to fit the linear regression model and to process data for quantifying the gain of the VOR. MS, JB and NT evaluated the data, supervised the data analyses and wrote the manuscript. All authors read and approved the final manuscript.

\section{Acknowledgements}

This work was supported by grants from the Cotrel Foundation Institut de France (MS, NT and JB) and NSERC discovery grant program and collaborative health research projects to MS and NT. The authors thank MarieFrance Dupuis for her help with Fig. 3, the staff at the Clinique infantile de Québec for the administrative support and the participants and parents for their time.

\section{References}

I. Herman R, Mixon J, Fisher A, Maulucci R, Stuyck J: Idiopathic scoliosis and the central nervous system: a motor control problem. The Harrington lecture, 1983. Scoliosis Research Society. Spine 1985, I O(I): I-I4.

2. Nault ML, Allard P, Hinse S, Le Blanc R, Caron O, Labelle H, Sadeghi $\mathrm{H}$ : Relations between standing stability and body posture parameters in adolescent idiopathic scoliosis. Spine 2002, 27(17): $1911-1917$.

3. Lao ML, Chow DH, Guo X, Cheng JC, Holmes AD: Impaired dynamic balance control in adolescents with idiopathic scoliosis and abnormal somatosensory evoked potentials. J Pediatr Orthop 2008, 28(8):846-849.

4. Guo X, Chau WW, Hui-Chan CW, Cheung CS, Tsang WW, Cheng JC: Balance control in adolescents with idiopathic scoliosis and disturbed somatosensory function. Spine 2006, 3 I (14): E437-440.

5. Machida M, Dubousset J, Imamura Y, Iwaya T, Yamada T, Kimura J, Toriyama S: Pathogenesis of idiopathic scoliosis: SEPs in chicken with experimentally induced scoliosis and in patients with idiopathic scoliosis. J Pediatr Orthop 1994, I 4(3):329-335.

6. Simoneau M, Mercier P, Blouin J, Allard P, Teasdale N: Idiopathic scoliosis changes the sensory-weighting mechanisms involved in balance control. BMC Neuroscience 2006, $19(7): 68$.

7. Sahlstrand T, Ortengren R, Nachemson A: Postural equilibrium in adolescent idiopathic scoliosis. Acta Orthop Scand 1978, 49(4):354-365.

8. ByI NN, Gray JM: Complex balance reactions in different sensory conditions: adolescents with and without idiopathic scoliosis. J Orthop Res 1993, I I(2):2 I5-227.

9. Driscoll DM, Newton RA, Lamb RL, Nogi J: A study of postural equilibrium in idiopathic scoliosis. J Pediatr Orthop 1984, 4(6):677-68I.

10. Simoneau M, Richer N, Mercier P, Allard P, Teasdale N: Sensory deprivation and balance control in idiopathic scoliosis adolescent. Exp Brain Res 2006, I70(4):576-582.

II. Gauchard GC, Lascombes P, Kuhnast M, Perrin PP: Influence of different types of progressive idiopathic scoliosis on static and dynamic postural control. Spine 200I, 26(9): I052-1058.

12. Fasold O, von Brevern M, Kuhberg M, Ploner CJ, Villlinger A, Lempert T, Wenzel R: Human vestibular cortex as identified with caloric stimulation in functional magnetic resonance imaging. Neuroimage 2002, I7(3): 1384-1393.

13. Ventre-Dominey J, Vallee B: Vestibular integration in human cerebral cortex contributes to spatial remapping. Neuropsychologia 2007, 45(2):435-439. 
14. Ivanenko YP, Grasso R, Israel I, Berthoz A: The contribution of otoliths and semicircular canals to the perception of twodimensional passive whole-body motion in humans. J Physiol I997, 502(Pt I):223-233.

15. Blouin J, Vercher JL, Gauthier G, Paillard J, Bard C, Lamarre Y: Perception of whole-body rotations in the absence of neck and body proprioception. J Neurophysiol 1995, 745:2216-2219.

16. Berthoz A, Israel I, Vieville T, Zee D: Linear head displacement measured by the otoliths can be reproduced through the saccadic system. Neurosci Lett 1987, 82(3):285-290.

17. Bloomberg J, Jones GM, Segal B, McFarlane S, Soul J: Vestibular-contingent voluntary saccades based on cognitive estimates of remembered vestibular information. Adv Otorhinolaryngol 1988, 4I:7|-75.

18. Blouin J, Gauthier GM, Vercher JL: Visual object localization through vestibular and neck inputs. 2: Updating off-mid-sagittal-plane target positions. J Vestib Res 1997, 7(23): |37-|43.

19. Israel I, Sievering D, Koenig E: Self-rotation estimate about the vertical axis. Acta Otolaryngol (Stockh) 1995, I I 5( I):3-8.

20. Ventre-Dominey J, Vighetto A, Denise P: Vestibulo-ocular dysfunction induced by cortical damage in man: a case report. Neuropsychologia 1999, 37(6):7I5-72I.

21. Ventre-Dominey J, Nighoghossian N, Denise P: Evidence for interacting cortical control of vestibular function and spatial representation in man. Neuropsychologia 2003, 4 I (I 4): I884- I898.

22. Israel I, Rivaud S, Gaymard B, Berthoz A, Pierrot-Deseilligny C: Cortical control of vestibular-guided saccades in man. Brain 1995 , | | 8(Pt 5): | | 69- | |83.

23. Pierrot-Deseilligny C, Rivaud S, Gaymard B, Agid Y: Cortical control of memory-guided saccades in man. Exp Brain Res 1991, 83:607-617.

24. Liu T, Chu WC, Young G, Li K, Yeung BH, Guo L, Man GC, Lam WW, Wong ST, Cheng JC: MR analysis of regional brain volume in adolescent idiopathic scoliosis: neurological manifestation of a systemic disease. J Magn Reson Imaging 2008, 27(4):732-736.

25. Seemungal BM, Rizzo V, Gresty MA, Rothwell JC, Bronstein AM Cortical processing in vestibular navigation. Prog Brain Res 2008, I 7 1:339-346.

26. Moreau A, Wang DS, Forget S, Azeddine B, Angeloni D, Fraschini F, Labelle H, Poitras B, Rivard CH, Grimard G: Melatonin signaling dysfunction in adolescent idiopathic scoliosis. Spine 2004, 29(16): |772-178|.

27. Fauteck JD, Lerchl A, Bergmann M, Moller M, Fraschini F, Wittkowski W, Stankov B: The adult human cerebellum is a target of the neuroendocrine system involved in the circadian timing. Neurosci Lett 1994, I 79( I 2):60-64.

28. Angelaki DE, Hess BJ: Inertial representation of angular motion in the vestibular system of rhesus monkeys. II. Otolith-controlled transformation that depends on an intact cerebellar nodulus. J Neurophysiol I995, 73(5):|729-175I.

29. Bronstein AM, Grunfeld EA, Faldon M, Okada T: Reduced selfmotion perception in patients with midline cerebellar lesions. Neuroreport 2008, 19(6):69|-693.

30. Buttner U, Fuchs AF, Markert-Schwab G, Buckmaster P: Fastigial nucleus activity in the alert monkey during slow eye and head movements. I Neurophysiol 1991, 65(6):1360-1371.

31. Manzoni D, Miele F: Vestibular mechanisms involved in idiopathic scoliosis. Arch Ital Biol 2002, I40(I):67-80.

32. Pompeiano $O$, Manzoni D, Miele F: Pineal gland hormone and idiopathic scoliosis: possible effect of melatonin on sleeprelated postural mechanisms. Arch Ital Biol 2002 I 40(2): | 29-I 58.

33. Uemura T, Arai Y, Skimazaki C: Eye-head coordination during lateral gaze in normal subjects. Acta Otolaryngologia 1980, 90:191-198

34. Blouin J, Labrousse L, Simoneau M, Vercher JL, Gauthier GM: Updating visual space during passive and voluntary head-in-space movements. Exp Brain Res 1998, 122:93-100.

35. Collewijn H, Smeets JB: Early components of the human vestibulo-ocular response to head rotation: latency and gain. I Neurophysiol 2000, 84(I):376-389.

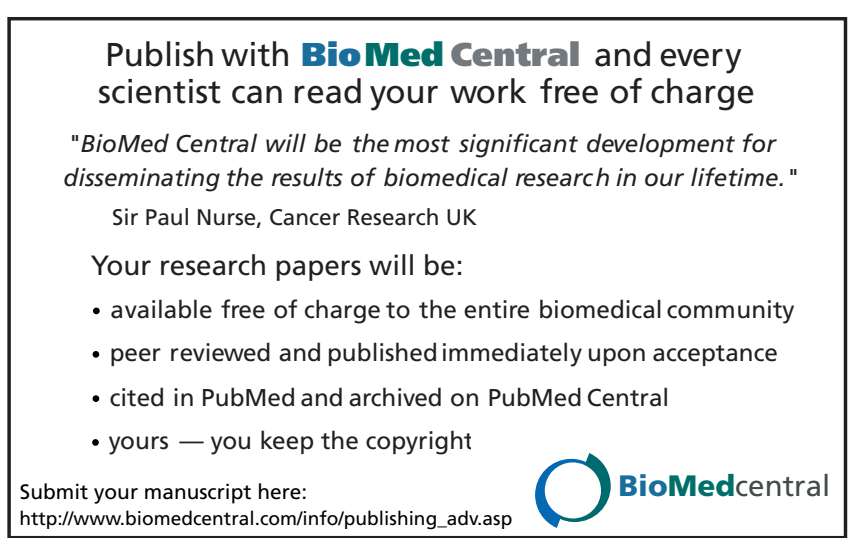

